\title{
Substorm-time magnetic field perturbations in the polar magnetosphere: POLAR observations
}

\author{
H. Kawano ${ }^{1}$, G. Le ${ }^{2}$, C. T. Russell ${ }^{3}$, G. Rostoker ${ }^{4}$, M. J. Brittnacher ${ }^{5}$, and G. K. Parks ${ }^{6}$ \\ ${ }^{1}$ Department of Earth and Planetary Sciences, Kyushu University, Fukuoka City, Fukuoka 812-8581, Japan \\ ${ }^{2}$ Laboratory for Extraterrestrial Physics, NASA/Goddard Space Flight Center, Greenbelt, MD 20771, U.S.A. \\ ${ }^{3}$ Institute of Geophysics and Planetary Physics, University of California, Los Angeles, CA 90095-1567, U.S.A. \\ ${ }^{4}$ Department of Physics, University of Alberta, Edmonton, AB T6G2J1, Canada \\ ${ }^{5}$ Geophysics Program, University of Washington, Seattle, WA 98195-1650, U.S.A. \\ ${ }^{6}$ Space Sciences Laboratory, University of California, Berkeley, CA 94720, U.S.A.
}

(Received April 30, 2002; Revised August 22, 2002; Accepted September 9, 2002)

\begin{abstract}
We present a case study of substorm-time magnetic field perturbations in the high-altitude polar magnetosphere, corresponding to the polar lobe, using data from the POLAR spacecraft together with ground-based CANOPUS observations and WIND solar-wind observations. The substorm of this paper had a clear growth phase and one clear major expansion onset, enabling an unmistakable comparison of the substorm timings and those of the magnetic field perturbations observed by POLAR. During the growth phase, the magnetic field strength $\left(B_{\text {total }}\right)$ increased and the flaring angle of the field line decreased at POLAR: They are ascribed to the pileup of magnetic field lines over the polar magnetosphere and to the braking effect of the ionosphere on the tailward motion of the piled-up field lines, respectively. For $\sim 28 \mathrm{~min}$ after the expansion onset, $B_{\text {total }}$ at POLAR did not decrease toward its presubstorm value, in contrast to past tail-lobe observations where $B_{\text {total }}$ decreased during the expansion phase. This absence of the field-decrease signature in the polar lobe for $\sim 28 \mathrm{~min}$, reported for the first time in this paper, could be ascribed to dipolarization/compression of the inner magnetosphere during the expansion phase, cancelling the field-decreasing effect.
\end{abstract}

\section{Introduction}

With its long dwell time in high-altitude polar magnetosphere, POLAR enables for the first time a detailed in-situ study of substorm-time perturbations in the polar magnetosphere. As an initial report, Kawano et al. (1997) (referred to as K97 below) presented a case in which the POLAR spacecraft stayed near the apogee in the polar magnetosphere during a substorm. The time sequence of the substorm itself was mainly monitored by ground magnetometers located near the local midnight meridian. POLAR observed an increase in the magnetic field strength during the growth phase of the substorm, then a recovery toward the pre-substorm level during the expansion phase. This is basically the same as the pattern observed in the further-downtail lobe (e.g. Russell and McPherron, 1973).

However, the complication was that the event studied by K97 had three expansion onsets over $\sim 52 \mathrm{~min}$, each with a comparable magnitude, so there existed uncertainties in the above-stated time correspondence. In addition, the interplanetary magnetic field (IMF) $B_{Z}$ of the event was such that the magnetic field strength at POLAR increased while the IMF $B_{Z}$ was negative, then started to recover around the time the IMF $B_{Z}$ turned toward positive. That is, it was possible that the field strength change at POLAR simply reflected

Copy right(c) The Society of Geomagnetism and Earth, Planetary and Space Sciences (SGEPSS); The Seismological Society of Japan; The Volcanological Society of Japan; The Geodetic Society of Japan; The Japanese Society for Planetary Sciences. the change in the energy input from the solar wind via the dayside reconnection. Furthermore, POLAR carries auroral imagers, which are very useful in capturing the global substorm sequence, but they were not operating during the event studied by K97.

In this paper we present another substorm event which had a simpler time sequence with one major expansion onset, as monitored by an auroral imager on board POLAR. The IMF $B_{Z}$ was continuously negative till the end of the substorm, in contrast to the event of $\mathrm{K} 97$ where the IMF $B_{Z}$ turned positive at the expansion onset time.

\section{Observations}

Figure 1 shows the magnetic field observed by the MFE instrument on board POLAR (Russell et al., 1995) on Apr. 21, 1996 along with simultaneous solar wind data (Lepping et al., 1995; Ogilvie et al., 1995) and the key parameter CL derived using ground magnetometer data from CANOPUS (Rostoker et al., 1995) that indicates the strength of the westward electrojet flowing over the stations of the array. The array extends over $\sim 45$ degrees of longitude, with the northsouth line of stations through Fort Churchill positioned at its eastern end at a geographic longitude of $\sim 265$ degrees; for the interval of Fig. 1, the local time of the Churchill line changed from 23:00 to 05:00 so that it was well placed to monitor the westward electrojet during our event shown in the figure.

The orbit of POLAR for the interval of Fig. 1 is shown in 


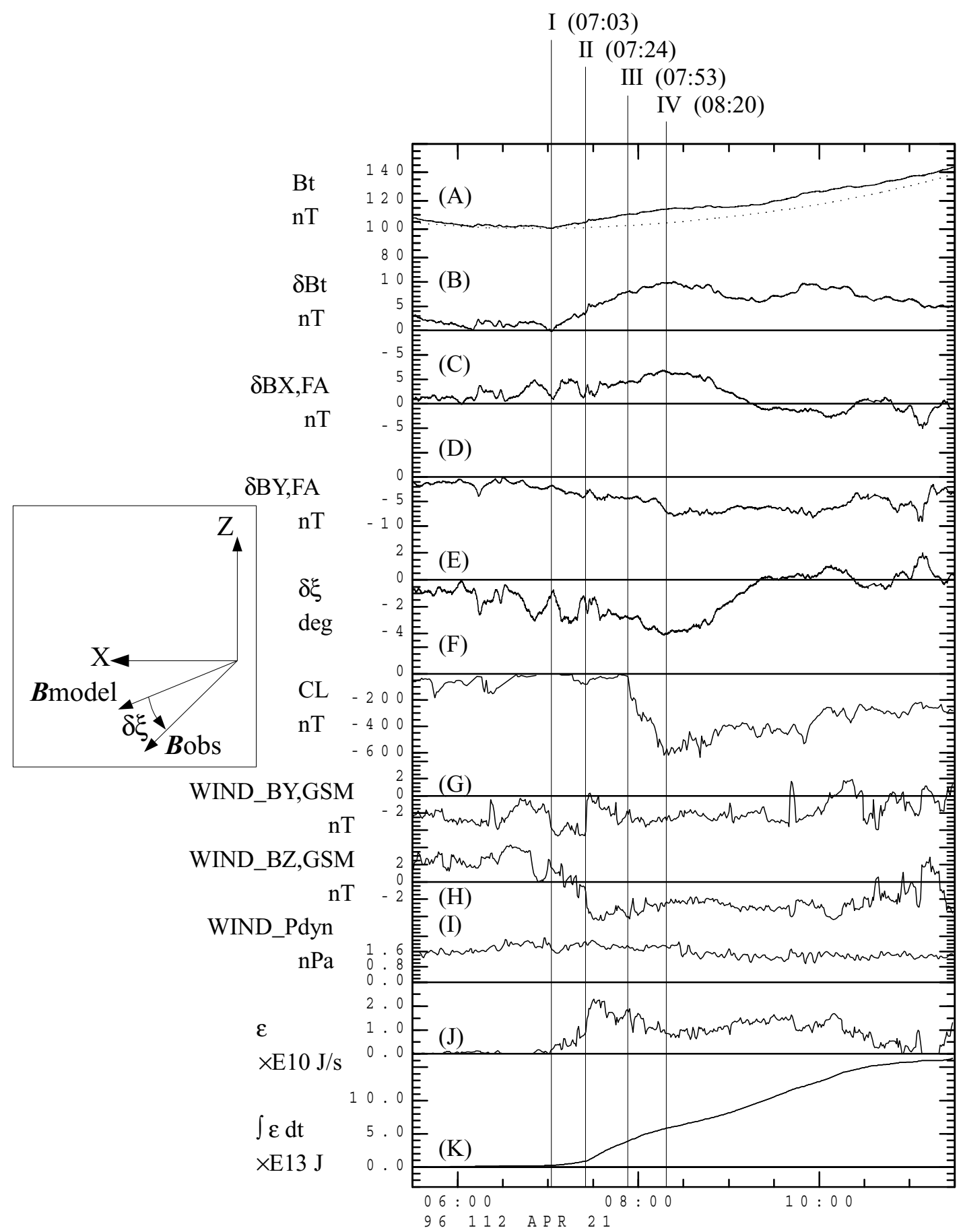

Fig. 1. Shows, from top, the magnetic field data observed by POLAR (Panels A-E), the key parameter CL from the CANOPUS network (F), the IMF $B_{Y}$ and $B_{Z}$ (in GSM coordinates) (Panels G-H), the solar wind dynamic pressure observed by WIND (Panel I), Perreault and Akasofu's (1978) $\epsilon$ parameter as a proxy measure of the solar wind energy input into the magnetosphere (Panel $\mathrm{J}$ ) and its time integral $\int \epsilon d t$ (Panel $\mathrm{K}$ ). The horizontal axis shows the universal time (UT). The propagation lag in Panels G-I is already roughly corrected by adding $-V_{X} / X$ to the observed time, where $V_{X}$ and $X$ are the GSM $X$ components of the solar wind ion bulk velocity observed by WIND and the WIND position, respectively. Panel A shows the total magnetic field; the solid line shows the observed values, and the dotted line shows the Tsyganenko (1996) model values. Panel B shows the difference of the two lines in the top panel. Refer to text for more detail and for the explanation of Panels C and D. Also refer to text for Panel E and the insert at the leftside edge of the figure. Vertical line I is drawn at the time (07:03 UT, as shown at the right-hand side of the character 'I') after which the IMF was horizontal then continuously southward; after this time $\epsilon$ (Panel J) was non-negligible. Vertical line II is drawn at the time (07:24 UT) after which the increase in $\epsilon$ (Panel J) was rapid, caused by the decrease and increase in the magnitudes of negative IMF $B_{Y}\left(\right.$ Panel G) and negative IMF $B_{Z}$ (Panel $\left.\mathrm{H}\right)$. Vertical line III is drawn at the time (07:53 UT) when $|\mathrm{CL}|$ (Panel F) started to expansively increase. Vertical line IV is drawn at the time (08:20 UT) when $\delta B_{\text {total }}$ (Panel B) and $\delta \xi$ (Panel E) at POLAR reached a maximum. 
Fig. 2: The figure shows that POLAR stayed in high-altitude polar magnetosphere, corresponding to the polar lobe, for the entire interval of Fig. 1, which includes the substorm of this paper.

We first examine the solar wind data. Their propagation lag is already roughly corrected by adding $-V_{X} / X$ to the observed time, where $V_{X}$ and $X$ are the GSM $X$ components of the solar wind ion bulk velocity observed by WIND and the WIND position, respectively. The position of WIND in GSM coordinates was $(34.5,-32.1,-8.1) R_{E}$ at $05: 30$ UT and $(38.2,-33.0,-3.2) R_{E}$ at 11:30 UT.

Panel I of Fig. 1 shows that the solar wind dynamic pressure, $P_{\text {dyn }}$, was fairly constant throughout the interval of the figure. On the other hand, the IMF $B_{Z}($ Panel $\mathrm{H})$ changed its sign from $\sim 06: 48 \mathrm{UT}$ to $\sim 07: 30 \mathrm{UT}$. That is, $B_{Z}$ was continuously positive for more than one hour before $\sim 06: 48 \mathrm{UT}$, when it started to decrease. Oscillations were superposed on the decrease in $B_{Z}$ from $\sim 06: 48$ UT to $\sim 07: 24$ UT (vertical line II), but after that time $B_{Z}$ monotonically decreased and reached a minimum at $\sim 07: 30 \mathrm{UT}$. From that time, $B_{Z}$ was negative and fairly constant for about three hours.

We note here that the GEOTAIL spacecraft was also located in the solar wind: Its position in GSM coordinates was $(10.63,27.72,5.37) \quad R_{E}$ at $05: 30$ UT and $(7.46,29.21,1.39) R_{E}$ at 11:30 UT. That is, GEOTAIL was at $Y \simeq 30$ while WIND was at $Y \simeq-30$, and the above-
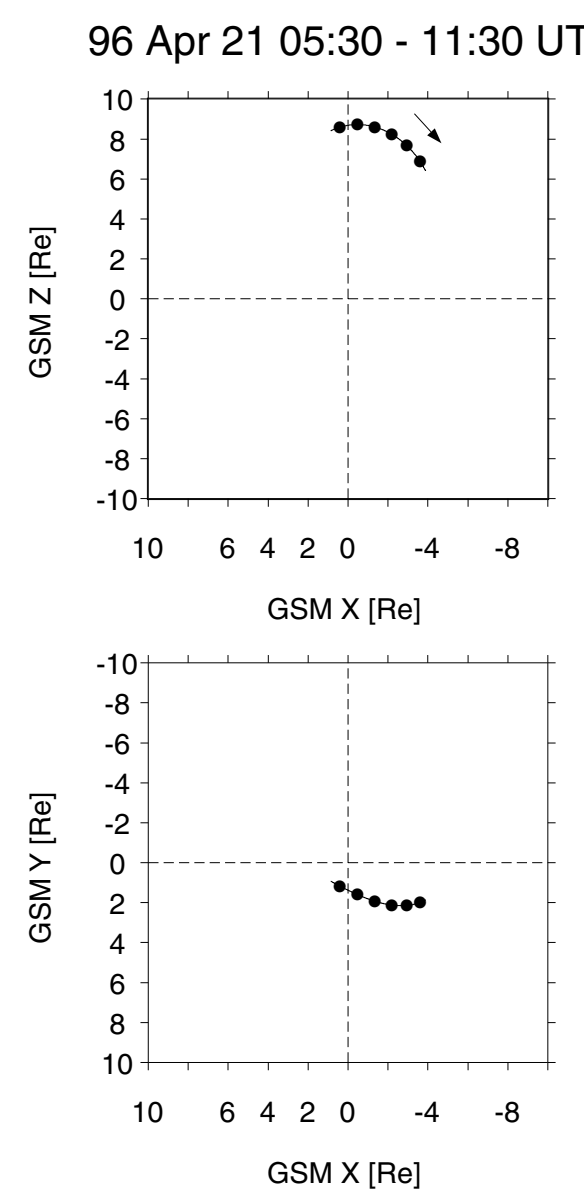

Fig. 2. The orbit of POLAR in GSM coordinates. Solid circles are drawn every hour. The direction of the satellite motion is indicated by the arrow in the top panel. stated signatures observed by WIND were also observed by GEOTAIL (not shown). Thus we are certain that the WIND data shown here significantly represent the solar wind encountered by the magnetosphere.

Panel J shows Perreault and Akasofu's (1978) $\epsilon$ parameter (calculated from the WIND data), which is a proxy measure of the solar wind energy input into the magnetosphere. After the vertical line II, i.e., when the IMF $B_{Z}$ (Panel H) was negative, the time profile of $\epsilon$ was similar (if one vertically inverts it) to that of the IMF $B_{Z}$. Before the vertical line II, $\epsilon$ started to increase at $\sim 07: 03$ UT (vertical line I), although the IMF $B_{Z}$ was small and positive on average between the lines I and II; its main cause was a simultaneous increase in the magnitude of the IMF $B_{Y}$ (Panel G), changing the IMF direction from northward to horizontal. Before the vertical line I, $\epsilon$ was negligibly small, corresponding to the positive sign of the IMF $B_{Z}$.

Panel K shows the time integral of the $\epsilon$ parameter, $\int \epsilon d t$; the start time of the integral is the start time of the figure (05:30 UT). This quantity will be discussed in Section 3.3 along with Panel B.

The CL parameter (Panel F) started to suddenly enhance at $\sim 07: 53$ UT (vertical line III). $|\mathrm{CL}|$ reached a maximum at 08:17 UT (close to the vertical line IV), followed by a gradual decrease toward the quiet level.

We also have auroral images for this event, both from the sky and the ground. Figure 3 shows auroral images taken by the UVI instrument on board POLAR (Torr et al., 1995). UVI acquires an image every $37 \mathrm{~s}$, and Fig. 3 shows selected UVI images in the interval 07:25-07:55 UT. Figure 3 shows that the nightside auroral oval moved equatorward till $\sim 07: 52$ UT, when the premidnight portion of the oval started to brighten and expand as an auroral bulge. The full set of 37-s UVI images (not all shown) indicates that the brightening started between 07:51:08 and 07:51:45 UT. The center of the bulge was located near 22:40 MLT. (Due to the POLAR platform wobble, the spatial resolution of the UVI image has been degraded to 1 by 10 pixels, but we do not expect an instrumental bias in the location of the center of the bulge.)

Figure 4 shows the meridional scanning photometer data taken at a CANOPUS station Fort Smith. It is a time-series plot and thus clearly shows the time-dependent latitudinal motion of the auroral oval, even though it lacks longitudinal information. The vertical line in Fig. 4 is drawn at the same time as the vertical line I in Fig. 1, i.e., at the start time of the solar wind energy input into the magnetosphere (see Panel J of Fig. 1). Figure 4 shows that the oval was stationary before the time of the vertical line, then started moving equatorward. At $\sim 07: 53$ UT (vertical line III in Fig. 1), the oval suddenly brightened and its poleward edge rapidly moved poleward. The observing station Fort Smith was located at 23:17 MLT at 07:53 UT.

Finally, we present the magnetic field data observed by POLAR. The solid curve in Panel A of Fig. 1 shows the observed total magnetic field, $B_{\text {total }}$. The curve shows a smoothly decreasing-then-increasing long-term trend, and superposed to it a perturbation starting at the vertical line I, as shown by a bent at the line I. (Note that the line I is drawn where the solar wind energy input started to increase (Panel J); thus it is natural that corresponding perturbation in 


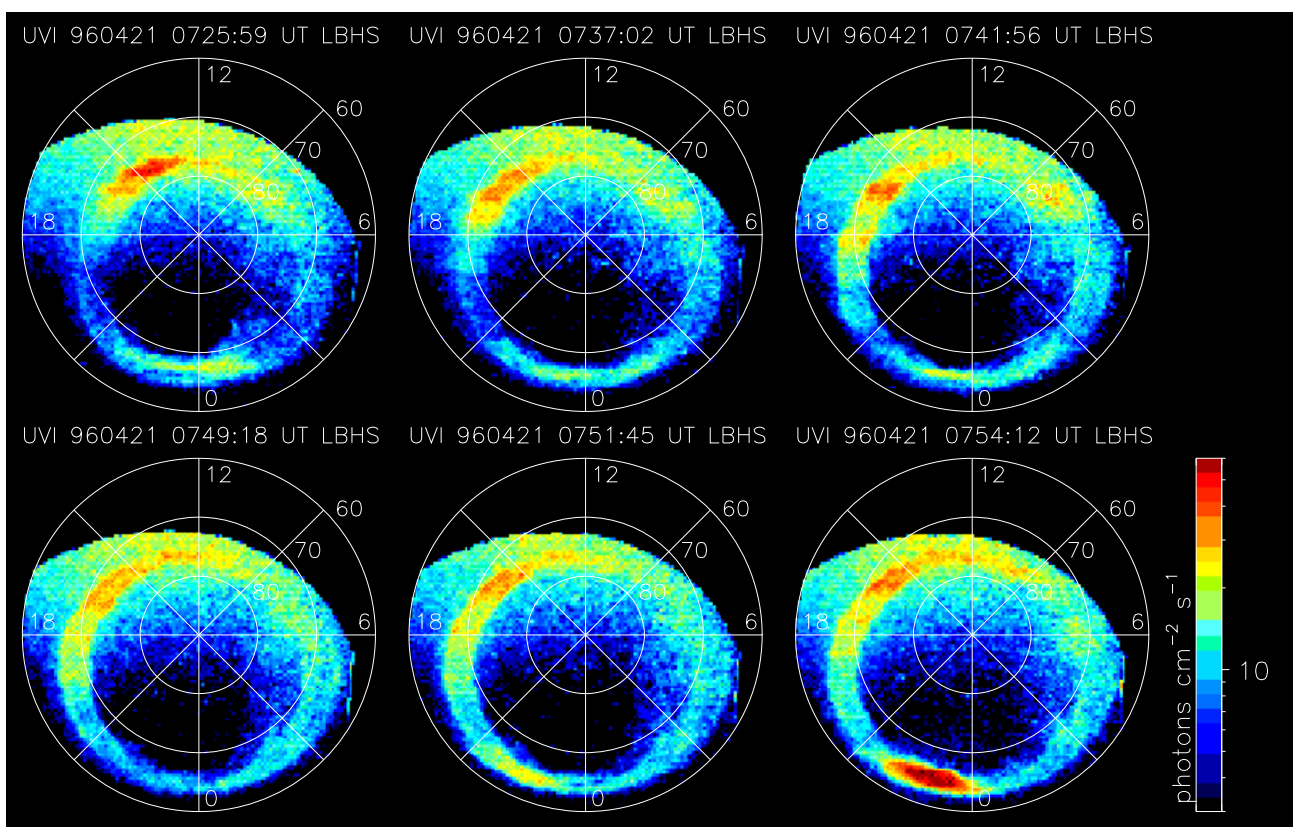

Fig. 3. Auroral images observed by the UVI instrument on board POLAR. Time of each panel is shown at the top-right side of the panel. As shown in each panel, the up direction of the panel corresponds to 12 hour MLT, and the down direction corresponds to 24 hour MLT.

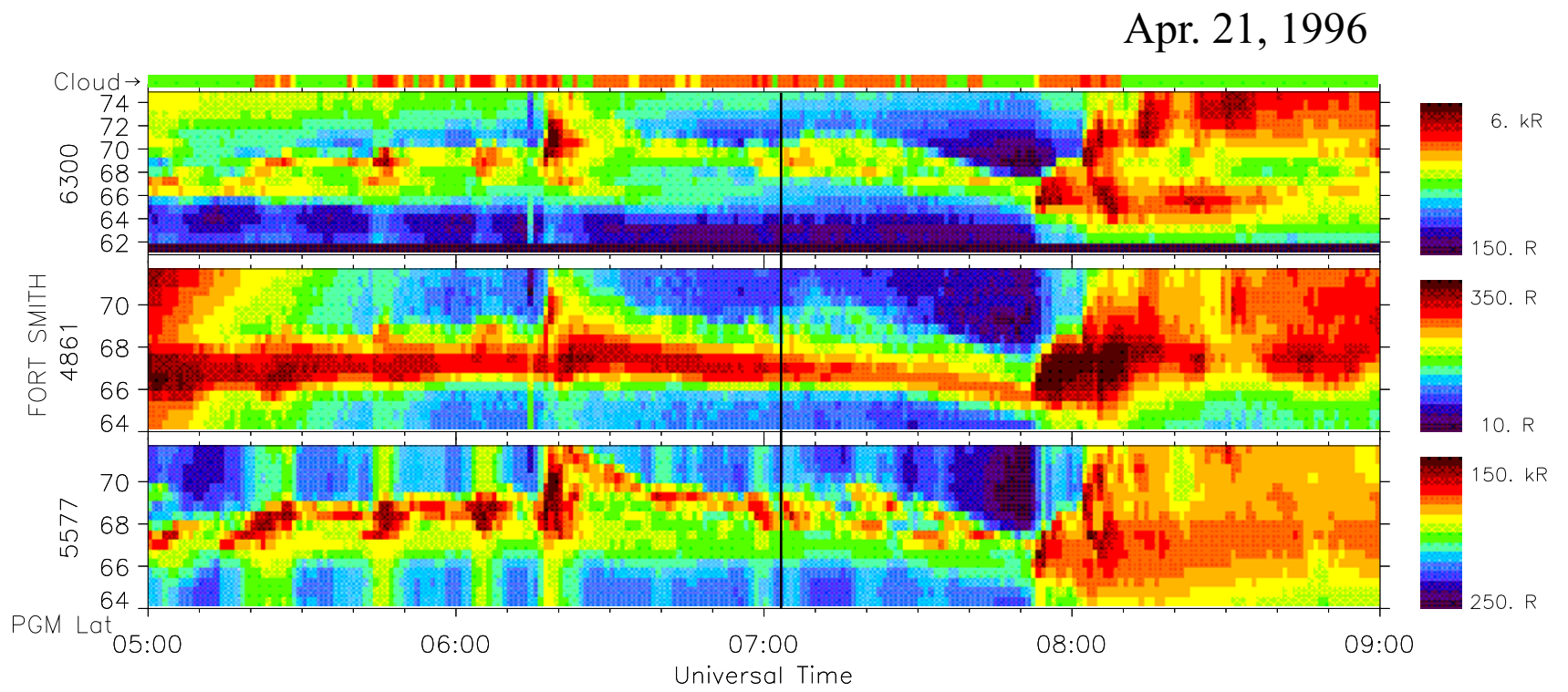

Fig. 4. Time series plots of the scanning photometer data at a CANOPUS station Fort Smith. The three panels show, from top, $630.0 \mathrm{~nm}, 486.1 \mathrm{~nm}$, and $557.7 \mathrm{~nm}$ wavelength data. The vertical axis of each panel is the latitude in degree. The superposed vertical line is drawn at the same time as the vertical line I in Fig. 1.

$B_{\text {total }}$ started at POLAR at that time.)

The smoothly decreasing-then-increasing long-term trend in $B_{\text {total }}$ is not a time-dependent change in the field but an apparent effect of the satellite motion (Fig. 2); we want to remove it and extract the true time-dependent perturbation in $B_{\text {total }}$. For this purpose, we use the Tsyganenko (1996) model as a model expressing the position dependence of the field, and subtract its value at the satellite position from the observed data. For this purpose the model itself should not be time dependent, so we fix the model parameters: For each of the model parameters, we use the average of its observed values during the interval 05:30-06:30 UT, for which all the model parameters were fairly constant. That is, $P_{\text {dyn }}=1.6 \mathrm{nPa}$ (Panel I), IMF $B_{Y}=-2.6 \mathrm{nT}$ (Panel G), IMF $B_{Z}=2.4 \mathrm{nT}$ (Panel H), and Dst $=-17.5 \mathrm{nT}$. The corresponding model field strength at the satellite position is shown in Panel A as the dotted curve.

(We note that we have tried a few other sets of values as the fixed model parameters, and we have found that the observed-minus-model field, such as $\delta B_{\text {total }}$ and $\delta \xi$ below, are essentially the same except for constant offsets (not shown). That is, the results shown below are essentially un- 
affected by the values of the fixed model parameters.)

Panel B shows $\delta B_{\text {total }}$, the observed field strength (solid curve in Panel A) minus the above-explained model field strength (dotted curve in Panel A): As explained above, this $\delta B_{\text {total }}$ shows the true time-dependent perturbation in the magnetic field strength in the polar magnetosphere. As expected, the perturbation starting at the vertical line I is clearly identified in $\delta B_{\text {total }}$.

Panels $\mathrm{C}$ and D show components of the observed minus model field vector in the field-aligned (FA) coordinate system. Here, the $Z_{\mathrm{FA}}$ unit vector $\mathbf{e}_{\mathrm{z}, \mathrm{FA}}$ is parallel to $\mathbf{B}_{\text {model }}$, which refers to the above-explained model field vector, the $Y_{\mathrm{FA}}$ unit vector $\mathbf{e}_{\mathrm{y}, \mathrm{FA}}$ is defined to be parallel to $\mathbf{B}_{\text {model }} \times \mathbf{r}$, where $\mathbf{r}$ is the position vector of the spacecraft, and the $X_{\mathrm{FA}}$ unit vector $\mathbf{e}_{\mathrm{x}, \mathrm{FA}}$ completes a right-hand triad. For the interval of the figure, the spacecraft was located in the northern magnetosphere more or less close to the GSM $X Z$ plane (see Fig. 2), and $\mathbf{B}_{\text {model }}$ there is mainly southward and sunward. Thus $\mathbf{e}_{\mathrm{y}, \mathrm{FA}}$ was directed roughly dawnward, and $\mathbf{e}_{\mathrm{x}, \mathrm{FA}}$ was directed roughly sunward and northward. Panel $C$ shows $\delta B_{X, \mathrm{FA}}$, which is the component of the field difference vector along $\mathbf{e}_{\mathrm{x}, \mathrm{FA}}$, and Panel D shows $\delta B_{Y, \mathrm{FA}}$, which is the component along $\mathbf{e}_{\mathrm{y}, \mathrm{FA}}$. Figure 1 does not show the component along $\mathbf{e}_{\mathrm{z}, \mathrm{FA}}$, because it is almost identical to $\delta B_{\text {total }}$.

Panel E of Fig. 1 shows $\delta \xi \equiv \xi_{\text {obs }}-\xi_{\text {model }}$, where $\xi$ is the "field flaring angle" of the magnetic field in the GSM $X Z$ plane, which means the flaring angle of the magnetic field line at the point of the satellite. That is, in the northern hemisphere, the field flaring angle is zero if the field vector is parallel to $(1,0)$, where 1 is the $x$-component and 0 is the $z$ component; if the field vector is parallel to $(0,-1)$, the field flaring angle is $90^{\circ}$. We use this "field flaring angle" definition so that its time-dependent changes during the substorm can easily be compared with that of the magnetopause flaring angle.

$\xi_{\text {obs }}$ is $\xi$ of the observed field (not shown), and $\xi_{\text {model }}$ is $\xi$ of the above-explained model field (not shown). The reason for subtracting $\xi_{\text {model }}$ is, as in the case of $\delta B_{\text {total }}$ above, to remove the position-dependent apparent change in $\xi$. The insert at the leftside edge of Fig. 1 illustrates $\delta \xi: \mathbf{B}_{\text {model }}$ and $\mathbf{B}_{\text {obs }}$ in the insert refer to the model and observed field vectors projected onto the $X Z$ plane. As stated above, $\delta \xi$ is the angle between the two, and its sign is positive if one goes anticlockwise in the insert from $\mathbf{B}_{\text {model }}$ to $\mathbf{B}_{\text {obs }}$.

Around the time of the southward turning of the IMF (see Panel $\mathrm{H}$ and vertical line II), $\delta B_{\text {total }}$ (Panel B) started to increase and $\delta \xi$ (Panel E) started to decrease. To be more precise, $\delta B_{\text {total }}$ monotonically increased after $\sim 07: 03 \mathrm{UT}$ (vertical line I), which is also the time Perreault and Akasofu's $\epsilon$ parameter (Panel J) started to increase. $\delta \xi$ showed more complicated perturbations: It had two peaks between $\sim 06: 32$ UT and $\sim 07: 31 \mathrm{UT}$; perhaps the fluctuating IMF $B_{Y}$ and $B_{Z}$ at the same interval is causally related to them. After $\sim 07: 31 \mathrm{UT}$, the decrease in $\delta \xi$ was almost monotonic.

Both $\delta B_{\text {total }}$ and $\delta \xi$ reached a peak of their monotonic increase/decrease around 08:20 UT (vertical line IV). Then they recovered toward their values observed at $\sim 07: 03$ UT (vertical line I). However, $\delta B_{\text {total }}$ did not fully recover to the value at $\sim 07: 03$ UT but reached a minimum around 09:20 UT, then increased again, and reached another peak at $\sim 09: 50 \mathrm{UT}$. On the other hand, $\delta \xi$ fully recovered to the value at $\sim 07: 03$ UT and increased even more, monotonically in general, without a decrease corresponding to the increase in $\delta B_{\text {total }}$ for 09:20-09:50 UT.

\section{Discussion}

\subsection{Substorm phases}

The auroral brightening at $\sim 07: 52$ UT observed by the UVI instrument on board POLAR (Fig. 3) marks the expansion onset of a substorm. This expansion onset was also apparent in (almost) simultaneous enhancement in CL (Fig. 1, Panel F, vertical line III) and (almost) simultaneous auroral brightening observed by the scanning photometer at the CANOPUS station Fort Smith (Fig. 4). The small difference in the onset times $(\sim 1 \mathrm{~min})$ is likely to have emerged because the ground stations missed the exact point where the auroral breakup started.

We note that the IMF did not become northward simultaneously with the expansion onset and that there was no simultaneous sudden enhancement in $P_{\text {dyn }}$. One may consider this onset to be spontaneous (e.g., Henderson et al., 1996), although the fact that the IMF $B_{Z}$ had been changing to become less southward after $\sim 07: 38$ UT might be consistent with a triggered onset.

In Fig. 4, the equatorward motion of the auroral oval was apparent from $\sim 07: 03$ UT (vertical line, drawn at the same time as the vertical line I in Fig. 1) to $\sim 07: 53$ UT (the same time as the vertical line III in Fig. 1), where the former is the time after which the solar wind energy input into the magnetosphere was active (see Fig. 1, Panel J), and the latter is the above-stated expansion onset time. Thus it is clear that the interval from $\sim 07: 03$ UT to $\sim 07: 53$ UT (i.e., from vertical line I to III in Fig. 1) corresponds to the growth phase of this substorm.

Almost all substorms have multiple intensifications, but for this event, from inspection of the POLAR/UVI data (images after the $\sim 07: 52$ UT onset not shown here) and the magnetograms from the CANOPUS array (not shown here) we can definitely say that the scale size of the $\sim 07: 52$ UT intensification was larger than that of following intensifications. In further support of this, the GOES 9 satellite observed a dipolarization starting at $\sim 07: 52$ UT when it was located at $\sim 23 \mathrm{hr}$ MLT, but after that, it observed no major dipolarization onset till $\sim 10: 00$ UT (not shown here). Thus, the onset at $\sim 07: 52$ UT marks the beginning of large-scale energy release in the magnetosphere through the substorm expansion process.

\subsection{Possible ionospheric effect on the magnetic field change in the polar lobe during the substorm}

POLAR observed magnetic field perturbations in the polar lobe corresponding to this well-identified substorm. In general, $\delta B_{\text {total }}$ (field strength) monotonically increased and $\delta \xi$ (flaring angle of the magnetic field line) monotonically decreased during the growth phase. We interpret this behavior to be a result of the magnetic flux pileup over the polar magnetosphere. The piling-up magnetic flux was eroded from the dayside magnetosphere and then transported downtail with the solar wind. The increase in $\delta B_{\text {total }}$ is a direct result of the flux pileup.

On the other hand, the decrease in $\delta \xi$ (flaring angle of 
the magnetic field line) needs more consideration. One may think this behavior is opposite to what is expected, because the flaring angle of the magnetopause increases during the growth phase. Our explanation of this feature is as follows: As a result of the piling up of the magnetic flux, the quiettime polar magnetosphere is pushed inward and tailward (because the normal to the polar magnetopause is directed both inward and tailward). Thus the magnetic field lines in the polar magnetosphere also tend to move along the force, i.e., inward and tailward. However, the field lines run through the ionosphere, which tends to decelerate the tailward motion of the footprints of the field lines because of its conductivity. In other words, the field lines are more or less 'pinned' at the ionosphere, and still the field-line part far from the ionosphere continues to move tailward. As a result, the field line becomes less flared. Figure 5 illustrates this explanation.

Currents associated with the above plasma/field-line motion are as follows: At the ionospheric footpoints of the tailward-moving field lines, dawn-to-dusk Pedersen currents flow, because the motional-induction electric field, impinging on the ionosphere from the magnetosphere, is directed dawn-to-dusk. Then, at the dawnward and duskward edges

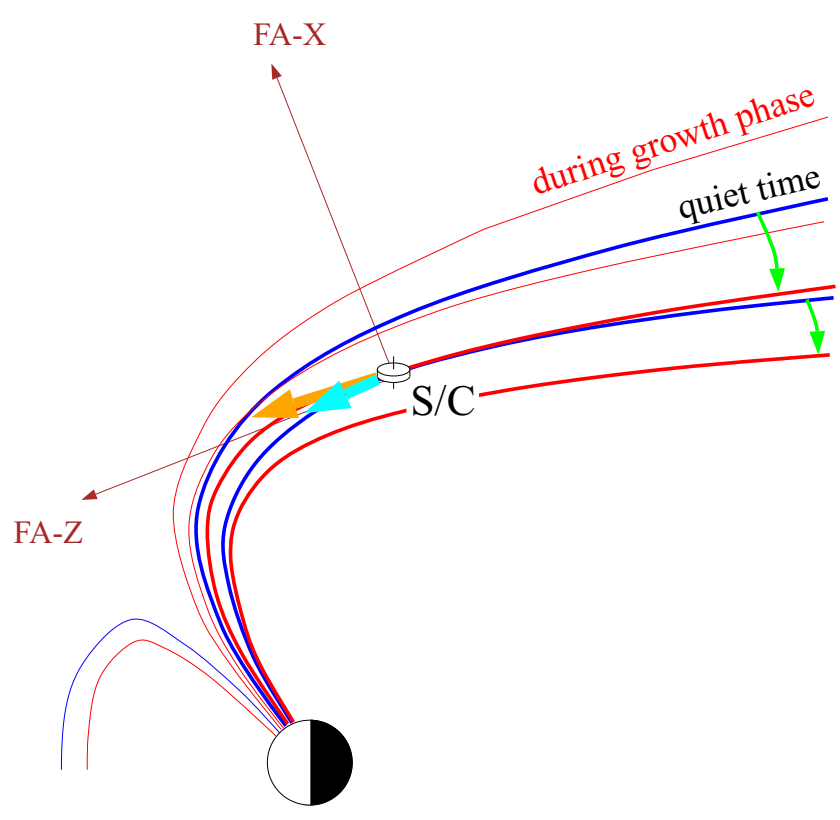

Fig. 5. Illustrates the motion and deformation of the magnetic field lines during the substorm growth phase. The blue lines correspond to the field lines during the quiet time. The red lines correspond to the field lines during the substorm growth phase. Motion and deformation of two blue lines toward the corresponding two red lines are indicated by two green arrows. Two thick arrows from the spacecraft (S/C in the figure) show the magnetic field vector observed by the spacecraft; the cyan arrow is that during the quiet time, and is tangent to the blue field line running through the spacecraft; the orange arrow is that during the growth phase, and is tangent to the red field line running through the spacecraft. Because of the compression due to the piled-up flux (represented by the outermost red field line), the field strength increases during the growth phase, as illustrated in the figure by the orange arrow longer than the cyan arrow. Because of the decelerating effect of the ionosphere (refer to text for details), the field line observed by the spacecraft becomes less flared during the growth phase, as illustrated in the figure by the orange arrow less tilted than the cyan arrow. Two thin arrows from the spacecraft show the directions of unit vectors in the field aligned coordinates (refer to text for its definition). of the ionospheric area where the tailward-moving field lines are mapped, the dawn-to-dusk Pedersen currents are connected to field-aligned currents whose sense is the same as the Region-1 currents. These field-aligned currents provide another way to explain the decrease in $\delta \xi$, equivalent to the above explanation in terms of the deceleration effect of the ionosphere, as follows: Between the two field-aligned currents (into the ionosphere in the dawnside, and out of the ionosphere in the duskside), sunward and northward-directed perturbation magnetic field is generated, and when it is added to the background field (sunward and southward), it decreases the field flaring angle. Note again that the two explanations are equivalent, because the ionospheric Pedersen conductivity is the source of the deceleration effect.

The deceleration effect of the ionosphere should exist, but it is difficult to quantitatively estimate how effective it is. As a future study, comparisons with the results of global MHD simulations should be worthwhile. The simulation scheme should properly include the ionosphere, of course. One could (artificially) change the ionospheric conductivity in the simulation code, cause a substorm, and check if the change in the field flaring angle is affected by the ionospheric conductivity.

\subsection{Apparent 28-min delay in the expansion onset in the polar lobe}

In the tail lobe, $\delta B_{\text {total }}$ reaches a maximum almost simultaneously with the substorm onset, and then recovers toward the pre-substorm value (e.g., Caan et al., 1978). On the other hand, in the event of this paper observed in the polar lobe, the maximum in $\delta B_{\text {total }}$ took place $\sim 28$ min after the expansion onset of the substorm (from vertical line III till IV in Fig. 1). This paper constitutes the first report of this delay.

In order to understand what caused this 28-min delay, below we first summarize factors which can affect $B_{\text {total }}$ in the polar lobe, and then discuss how they worked during the event of this paper. They are:

(1) Pileup of the dayside-reconnected magnetic flux;

(2) Rarefaction due to the reconnection at the near-Earth neutral line (NENL); and

\section{(3) Compression effect of the dipolarization;}

explanations of these three factors are given in the three paragraphs just below.

The above factor (1) is also observed in the tail lobe: The dayside-reconnected field lines pile up over the magnetosphere, including its polar region and its tail region. The amount of the piled-up flux is related to the efficiency of the dayside reconnection, and is approximately proportional to $\int \epsilon d t$ (Fig. 1, Panel K), which is a proxy measure of timeintegrated energy input from the solar wind to the magnetosphere through the dayside reconnection.

Factor (2) is also observed in the tail lobe: As the magnetic field lines are reconnected at NENL, the reconnected field lines are removed from the region northward and southward of NENL (and move to the region earthward and tailward of NENL). As a result, rarefaction takes place in the tail lobe region northward and southward of NENL. This rarefaction effect propagates, via the fast mode wave, to the lobe parts 
away from NENL, including the polar lobe where the POLAR satellite observed the event of this paper; as a result, $B_{\text {total }}$ in the polar lobe should start decreasing. Because the fast mode wave speed is very high $(>5000 \mathrm{~km} / \mathrm{s})$ in the lobe, $B_{\text {total }}$ in the polar lobe should start decreasing soon (in less than a minute) after the expansion onset.

Factor (3) does not operate in the tail lobe but operates in the polar lobe. It is well known that the magnetic field in the inner magnetosphere undergoes dipolarization after the expansion onset (see, e.g., Lopez et al., 1988, and references therein). It has been suggested that current disruption (CD below) causes the dipolarization Earthward of the CD region (see, e.g., Lui, 1996, and references therein). The dipolarization is the process in which field lines that were stretched during the growth phase recover toward their pregrowth-phase shape; Figure 6 illustrates it, in which arrows are drawn between two curves, showing the shape change of a field line after the expansion onset. (Note that the field-line shape after the expansion onset, more dipolar, is also similar to its shape before the start of the growth phase.) Figure 6 shows that, as a result of this dipolarization, the shaded parts of the field line move toward the polar lobe and compress it.

Currents generated during this dipolarization process are as follows. First, as is known well, dawn-to-dusk cross-tail currents are disrupted in the $\mathrm{CD}$ region. Then, CD causes dipolarization, which compresses the equatorial inner magnetosphere. The compressed equatorial inner magnetosphere exerts the pressure gradient force directed away from it; in the equatorial plane the force is directed tailward but it cannot win over the Earthward force from the CD region; on the other hand in the polar part (shaded part of Fig. 6), the force is directed poleward and moves the plasma/field line there poleward; this is the source of the compression of the polar lobe, including the magnetic field there. This increase in the polar lobe field is equivalent to additional dawn-to-dusk currents flowing near the shaded part of Fig. 6. These currents may be connected with field-aligned currents, but discussing the current closure is beyond the scope of this paper.

We note that Kistler et al. (1992) presented an idea similar to our factor (3): They observed cases of dipolarizationassociated compression at $7<r<10 R_{E}$ in the nightside

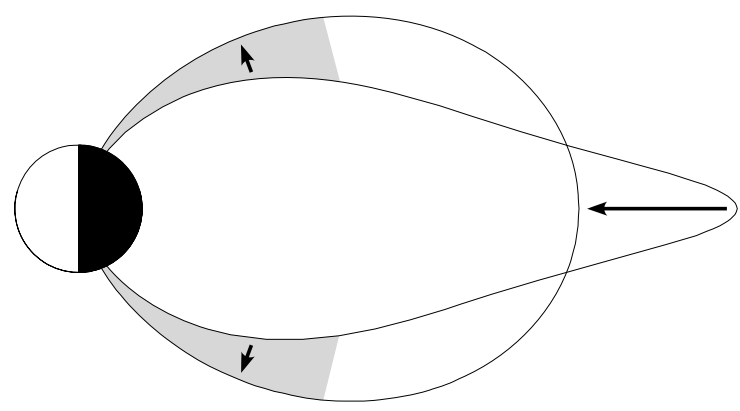

Fig. 6. Illustrates how the inner polar magnetosphere is compressed by dipolarization. Two lines in the figure show a field line before and after dipolarization. The field line motion as a result of the dipolarization is shown by arrows in the figure. The substorm onset region is located on the equatorial plane at or tailward (to the right in the figure) of the before-the-dipolarization field line. The shaded part in the figure shows a field line motion toward the polar magnetosphere, which compresses the polar magnetosphere. plasmasheet, where $r$ is the radial distance from the Earth. Based on this, even though they had data only in the equatorial magnetosphere, they predicted the field line topology in the entire magnetosphere before and after the expansion onset: See their Figure 9, which shows a field-topology change similar to that in our Fig. 6.

In relation to this, we note that Nakai and Kamide (1994) presented a magnetospheric current model that is consistent with the above observations by Kistler et al. (1992): The equatorial current in their model increases during the expansion phase in the inner magnetosphere, consistent with the compression of the inner magnetosphere owing to the dipolarization. This could also be regarded as a support of our factor (3).

We also note that, while CD leads to the increase in the field strength in the inner magnetosphere and the polar lobe via dipolarization (as stated above), CD leads to the decrease in the field strength in the tail lobe (e.g., Jacquey et al., 1991, 1993; Ohtani et al., 1992). However, this effect on the tail lobe field is not discussed below, because this paper is on the polar lobe field. For the polar lobe, CD leads to compression (factor (3)).

Having summarized the three factors above, we now discuss how the combination of these three factors could explain the observed behavior. As stated above, $\int \epsilon d t$ (Fig. 1, Panel K) approximates the factor (1). In Fig. 1 we see that the $\delta B_{\text {total }}$ profile (Panel B) is similar to the $\int \epsilon d t$ profile for the interval between the vertical lines I and IV. Thus, it is natural to assume that $\delta B_{\text {total }}$ in the interval between the vertical lines I and IV reflects the factor (1) only. On the other hand, the decrease in $\delta B_{\text {total }}$ after the vertical line IV can only be explained by the factor (2), because it is the only factor which decreases the field strength.

Then the question is, why did not the factor (2) show itself soon after the vertical line III = expansion onset? As stated above, it must become effective in the polar lobe in a minute after the expansion onset. However, the decrease in the observed $\delta B_{\text {total }}$ started at the vertical line IV, $\sim 28$ minutes after the onset.

As an answer to this question, one may think that the factor (2) was in fact effective in the polar lobe soon after the onset (vertical line III), but that the effect of the factor (1) may have further increased after the vertical line III, cancelling the effect of the factor (2). However, the above-stated similarity of the time profiles of $\delta B_{\text {total }}$ and $\int \epsilon d t$ before the vertical line IV already argues against this scenario. Put differently, if the effect of the factor (1) had further increased after the vertical line III, the slope of $\int \epsilon d t$ should have become more steep after that time, but the observed $\int \epsilon d t$ (Panel K) does not show it: It shows a constant-rate increase before and after the vertical line III.

Our answer to the question is, the factor (2) was in fact effective in the polar lobe soon after the onset (vertical line III), but the factor (3) balanced/cancelled it: In general, the factor (3) is operative after the expansion onset, and it increases the field strength. Thus, for our case, the summation of the factors (1) (increasing $\delta B_{\text {total }}$ ), (2) (decreasing $\delta B_{\text {total }}$ ), and (3) (increasing $\delta B_{\text {total }}$ ) should have contributed to $\delta B_{\text {total }}$ between the line III and IV, but as stated above, the actuallyobserved $\delta B_{\text {total }}$ reflects only the factor (1). Then the only 
possibility is that the factors (2) and (3) cancelled each other during the interval between the vertical lines III and IV.

The factor (2) became apparent in the observed $\delta B_{\text {total }}$ after the vertical line IV, when the expansion-phase activity started to decrease (note the CL parameter (Panel F) started to decrease in its magnitude then); this suggests that the activity of factor (3) was large only while the expansion-phase activity increased. This is very natural, because the dipolarization is the characteristic feature of the substorm expansion. That is, $\mathrm{CD}$ is active during the expansion phase, which causes the dipolarization and the current wedge, the latter of which leads to the enhancement in the ionospheric currents (thus CL); thus it is natural that the two occur concurrently.

In Subsection 3.2 we discussed the decrease in $\delta \xi$ during the growth phase, that is, between the vertical lines I and III. $\delta \xi$ continued to decrease between the vertical lines III and IV (Fig. 1, Panel E), which is not surprising because, in our scenario above, factor (1) was still dominant during that interval. On the other hand after the vertical line IV, $\delta \xi$ was observed to recover toward its pre-growth-phase value. This interval corresponds to the recovery phase, and our scenario above says factor (2) was dominant during this interval; this factor has the effect of returning the magnetosphere toward the pre-growth-phase state, including returning $\delta B_{\text {total }}$ toward its pre-growth-phase value. Thus, it is natural that factor (2) also returns $\delta \xi$ toward its pre-growth-phase value; otherwise the magnetosphere cannot return to its quite state, which is unrealistic. More detailed discussion of the $\delta \xi$ recovery belongs to future research: The main target of this paper is the growth and expansion phases.

Further examination of the delay needs a study of more substorms during a continuous southward IMF, which is a topic of future research. In relation to this, it is important to study whether the above factors (2) and (3) usually have comparable amplitudes during the expansion phase, as in the event of this paper. It is notable here that it is important to look at substorms which are not associated with the northward turning of the IMF, because the northward turning stops the factor (1), or differently put, causes a kink in the $\int \epsilon d t$ time profile at the onset time (unlike in our event), which makes it difficult to decompose the above-stated three factors.

To summarize this subsection, the apparent $\sim 28$-min delay of the expansion onset signature at POLAR in the polar lobe could be ascribed to cancellation of the following two contributions: Rarefaction of the polar lobe due to the reconnection at NENL (factor (2)), and the compression of the inner magnetosphere due to the dipolarization (factor (3)).

\subsection{Other features}

Another maximum in $\delta B_{\text {total }}$ (Fig. 1, Panel B) at $\sim 09: 50$ UT may correspond to another substorm. This idea is consistent with the behavior of $\mathrm{CL}$, which showed local minima at $\sim 09: 19, \sim 09: 29, \sim 09: 39$, and $\sim 09: 50$ UT: It is possible these local minima correspond to expansion onsets. Magnetometer data from a few CANOPUS ground stations show small and gradual enhancements in the Pi2-range wave activity around the above times (not shown here), which is also consistent with the idea of (small) onsets then. If the maximum in $\delta B_{\text {total }}$ at $\sim 09: 50$ UT actually corresponded to a substorm (with multiple onsets, perhaps), then it had an in- teresting feature: It was not associated with a decrease in $\delta \xi$. Our experience tells us that it is sometimes the case for the second (and later) one of recurring substorms, and it deserves to be statistically checked in the future.

$\delta B_{X \text {,FA }}$ (Panel C) and $\delta \xi$ (Panel E) are similar to each other if one vertically inverts one of them. This happened because the satellite was close to the GSM $X Z$ plane so that the magnetic field vector was nearly parallel to the $X Z$ plane. On the other hand, the perturbation in $\delta B_{Y, \mathrm{FA}}$ (Fig. 1, Panel D) is difficult to explain. Its pattern in this event is different from that in the K97 event, and it is difficult to find a common pattern. At least it appears unlikely that this component reflects fieldaligned currents during substorms. Perhaps $\delta B_{Y, \mathrm{FA}}$ does not have a special meaning for substorms in the polar magnetosphere, but it is just a mixture of $\delta B_{X, \mathrm{FA}}$ and $\delta B_{Z, \mathrm{FA}}$ : With a proper coordinate rotation, it may become flat.

Finally, we note that Russell (2000) has proposed an explanation for the often observed two stages of substorm expansion, before and after the time at which open lobe field lines start to be reconnected at NENL. (See Mishin et al. (2001) and references therein for the observations of the two stages; they report that the first-stage expansion lasts for 10 to 40 minutes.) In Russell's (2000) model, the second expansion phase takes place as a result of the northward turning of the IMF, because it stops the generation of closed field lines at the distant neutral line so that the open lobe field lines can reach the NENL. In the substorm of this paper, the IMF remained southward; perhaps reconnection stayed inside the plasma sheet and the reconnection rate remained small. In fact, POLAR/UVI images at times after those in Fig. 3 (not shown) indicate that the bulge forming at $\sim 07: 51$ UT (see Fig. 3) was the only major, lasting bulge. That is, there was no second-stage onset. Thus, substorms without a northward turning of the IMF may have different features from those with northward turning of the IMF.

\section{Summary}

In this paper we have studied a substorm with a clear growth phase and one clear major expansion onset. For this event the IMF $B_{Z}$ (southward) and the solar wind dynamic pressure, $P_{\text {dyn }}$, were fairly constant throughout the duration of the substorm. We have demonstrated that, at the site of POLAR which was located in the polar lobe, the magnetic field strength increased and the flaring angle of the magnetic field line decreased during the growth phase of the substorm, that they reached peak values $\sim 28 \mathrm{~min}$ after the expansion onset, and that they then recovered toward their pre-substorm values.

The increase in the field strength during the growth phase is ascribed to the pileup of magnetic field lines over the polar magnetosphere. The decrease in the field flaring angle during the growth phase is ascribed to the decelerating effect of the polar ionosphere over the tailward motion of field lines in the polar magnetosphere, caused by the pileup.

The $~ 28$-min delay from the expansion onset to the start of the recovery of the magnetic field in the polar lobe is reported for the first time in this paper with evidence that it was not caused by solar wind (IMF $B_{Z}$ and $P_{\text {dyn }}$ ) changes. "Why no onset effect for $\sim 28 \mathrm{~min}$ in the polar lobe" could be ascribed to the dipolarization/compression of the inner magne- 
tosphere after the expansion onset, cancelling the rarefaction effect of the reconnection at NENL; this compression effect lasted $\sim 28$ min after the expansion onset, during which the expansion-phase activity increased, as shown in the CL parameter.

We note that the multi-point, multi-instrument observations presented in this paper were impossible before, demonstrating the usefulness of the international collaboration in the ISTP era. It is important that the features reported in this paper are confirmed by a statistical study; it is also important to compare the observations with results of global MHD simulations to examine our explanations for the observations; these are subjects of future research.

Acknowledgments. The CANOPUS instrument array was constructed and is maintained and operated by the Canadian Space Agency for the Canadian scientific community. We appreciate K. Ogilvie for the data from WIND Solar Wind Experiment. We also appreciate R. Lepping for the data from Wind Magnetic Field Investigation. We also appreciate $\mathrm{S}$. Ohtani for his comments on this paper. G. Le and C. T. Russell were supported by the National Aeronautics and Space Administration (NASA) under research grant NAG5-7721. This work was partly supported by the Japan Society for the Promotion of Science under Grant-in-Aid for Scientific Research 13740286.

\section{References}

Caan, M. N., R. L. McPherron, and C. T. Russell, The statistical magnetic signature of magnetospheric substorms, Planet. Space Sci., 26, 269-279, 1978.

Henderson, M. G., G. D. Reeves, R. D. Belian, and J. S. Murphree, Observations of magnetospheric substorms occurring with no apparent solar wind/IMF trigger, J. Geophys. Res., 101, 10773-10791, 1996.

Jacquey, C., J. A. Sauvaud, and J. Dandouras, Location and propagation of the magnetotail current disruption during substorm expansion-analysis and simulation of an ISEE multi-onset event, Geophys. Res. Lett., 18, 389-392, 1991.

Jacquey, C., J. A. Sauvaud, J. Dandouras, and A. Korth, Tailward propagating cross-tail current disruption and dynamics of near-earth tail-A multipoint measurement analysis, Geophys. Res. Lett., 20, 983-986, 1993.

Kawano, H., C. T. Russell, G. Rostoker, T. Mukai, and H. Spence, Initial POLAR MFE observation of substorm signatures in the polar magnetosphere, Geophys. Res. Lett., 24, 1459-1462, 1997.

Kistler, L. M., E. Mobius, W. Baumjohann, and G. Paschmann, Pressure changes in the plasma sheet during substorm injections, J. Geophys. Res., 97, 2973-2983, 1992.
Lepping, R. P., M. H. Acuna, L. E. Burlaga, W. M. Farrell, J. A. Slavin, K. H. Schatten, F. Mariani, N. F. Ness, F. M. Neubauer, Y. C. Whang, J. B. Byrnes, R. S. Kennon, P. V. Panetta, J. Scheifele, and E. M. Worley, The WIND magnetic field investigation, Space Sci. Rev., 71, 207-229, 1995.

Lopez, R. E., A. T. Y. Lui, D. G. Sibeck, R. W. McEntire, L. J. Zanetti, T. A. Potemra, and S. M. Krimigis, The longitudinal and radial distribution of magnetic reconfigurations in the near-Earth magnetotail as observed by AMPTE/CCE, J. Geophys. Res., 93, 997-1001, 1988.

Lui, A. T. Y., Current disruption in the Earth's magnetosphere: Observations and models, J. Geophys. Res., 101, 13067-13088, 1996.

Mishin, V. M., T. Saifudinova, A. Bazarzhapov, C. T. Russell, W. Baumjohann, R. Nakamura, and M. Kubyshkina, Two distinct substorm onsets, J. Geophys. Res., 106, 13105-13118, 2001.

Nakai, H. and Y. Kamide, A model of the large-scale distribution of the neutral sheet current and its substorm-associated changes, J. Geophys. Res., 99, 2365-2373, 1994.

Ogilvie, K. W., D. J. Chornay, R. J. Fritzenreiter, F. Hunsaker, J. Keller, J. Lobell, G. Miller, J. D. Scudder, E. C. Sittler, Jr., R. B. Torbert, D. Bodet, G. Needell, A. J. Lazarus, J. T. Steinberg, J. H. Tappan, A. Mavretic, and E. Gergin, SWE, a comprehensive plasma instrument for the WIND spacecraft, Space Sci. Rev., 71, 55-77, 1995.

Ohtani, S., S. Kokubun, and C. T. Russell, Radial expansion of the tail current disruption during substorms - A new approach to the substorm onset region, J. Geophys. Res., 97, 3129-3136, 1992.

Perreault, P., and S.-I. Akasofu, A study of geomagnetic storms, Geophys. J. R. Astr. Soc., 54, 547-573, 1978.

Rostoker, G., J. C. Samson, F. Creutzberg, T. J. Hughes, D. R. McDiarmid, A. G. McNamara, A. V. Jones, D. D. Wallis, and L. L. Cogger, CANOPUS - a ground-based instrument array for remote sensing the high latitude ionosphere during the ISTP/GGS program, Space Sci. Rev., 71, 743-760, 1995.

Russell, C. T., How northward turnings of the IMF can lead to substorm expansion onsets, Geophys. Res. Lett., 27, 3257-3259, 2000.

Russell, C. T. and R. L. McPherron, The magnetotail and substorms, Space Sci. Rev., 11, 111-122, 1973.

Russell, C. T., R. C. Snare, J. D. Means, D. Pierce, D. Dearborn, M. Larson, G. Barr, and G. Le, The GGS/POLAR fields investigation, Space Sci. Rev., 71, 563-582, 1995.

Torr, M. R., D. G. Torr, M. Zukic, R. B. Johnson, J. Ajello, P. Banks, K. Clark, K. Cole, C. Keffer, G. Parks, B. Tsurutani, and J. Spann, A far ultraviolet imager for the International Solar-Terrestrial Physics mission, Space Sci. Rev., 71, 329-383, 1995.

Tsyganenko, N. A., Effects of the solar wind conditions on the global magnetospheric configuration as deduced from data-based field models, in Proceedings of the ICS-3 Conference on Substorms, Spec. Publ. SP389, pp. 181-185, European Space Agency, Paris, France, 1996.

H. Kawano (e-mail: hkawano@geo.kyushu-u.ac.jp), G. Le, C. T. Russell, G. Rostoker, M. J. Brittnacher, and G. K. Parks 